\title{
Role of Gut Microbiota in Type 2 Diabetes Mellitus and Its Complications: Novel Insights and Potential Intervention Strategies
}

\author{
Birhanu Woldeamlak, Ketsela Yirdaw ${ }^{1}$ and Belete Biadgo ${ }^{1}$ \\ Clinical Chemistry Laboratory, University of Gondar Hospital; Department of Clinical Chemistry, School of Biomedical and Laboratory Sciences, \\ College of Medicine and Health Sciences, University of Gondar ${ }^{1}$, Gondar, Ethiopia
}

\begin{abstract}
Type 2 diabetes mellitus has become one of the fastest growing public health problems worldwide. The disease is believed to involve a complex process involving genetic susceptibility and environmental factors. The human intestine harbors hundreds of trillions of bacteria, as well as bacteriophage particles, viruses, fungi, and archaea, which constitute a complex and dynamic ecosystem referred to as the gut microbiota. Increasing evidence has indicated changes in the gut microbiota composition or function in type 2 diabetic patients. An analysis of 'dysbiosis' enables the detection of alterations in the specific bacteria, clusters of bacteria, or bacterial functions associated with the occurrence of type 2 diabetes. These bacteria are involved predominantly in the control of inflammation and energy homeostasis. This review attempts to show that the gut microbiota are important factors for the occurrence of type 2 diabetes and are important for the treatment of gut microbiota dysbiosis through bariatric surgery, fecal microbiota transplantation, prebiotics, and probiotics. (Korean J Gastroenterol 2019;74:314-320)
\end{abstract}

Key Words: Type 2 diabetes mellitus; Gastrointestinal microbiome

\section{INTRODUCTION}

Diabetes mellitus (DM) is a metabolic disorder of multiple etiologies characterized by chronic hyperglycemia, resulting from deficiencies in insulin secretion, insulin action, or both. ${ }^{1}$ Type 2 DM (T2DM), which accounts for 90-95\% of diabetes cases, includes individuals with insulin resistance (IR) and frequently have relative insulin deficiency., ${ }^{1,2}$ Type 1 diabetes, which accounts for $10 \%$ of all cases, is characterized by an absolute deficiency of insulin secretion caused by pancreatic $\beta$-cell destruction, usually resulting from autoimmune attack. ${ }^{3}$

The prevalence of T2DM is increasing rapidly, presumably because of the increasing incidence of obesity, reduced physical activity levels as countries become more industrialized, and aging of the population. ${ }^{4}$ According to International Diabetes Federation, DM Atlas 2015 report, more than 75\% of people with DM live in low and middle income countries. ${ }^{5}$ In Africa, DM is expected to have the highest prevalence in the future, and the regional prevalence of DM has been reported to be $3.8 \%$ and is expected to increase to $4.3 \%$ in $2030 .^{6}$

The risk factors for DM include older age, obesity, family history of DM, prior history of gestational DM, impaired glucose tolerance, physical inactivity, and race/ethnicity. ${ }^{7}$ The human gut microbiota is a complex ecosystem, which is a key component in the gastrointestinal tract (GIT) homeostasis. The involvement of the gut microbiota in immune diseases has recently been demonstrated, and a bacterial imbalance

Received November 27, 2018. Revised January 9, 2019. Accepted January 23, 2019.

(C) This is an open access article distributed under the terms of the Creative Commons Attribution Non-Commercial License (http://creativecommons.org/licenses/ by-nc/4.0) which permits unrestricted non-commercial use, distribution, and reproduction in any medium, provided the original work is properly cited.

Copyright (C) 2019. Korean Society of Gastroenterology.

Correspondence to: Belete Biadgo, Department of Clinical Chemistry, School of Biomedical and Laboratory Sciences, College of Medicine and Health Sciences, University of Gondar, Po.box 196, Gondar, Ethiopia. Tel: +251-91-829-5071, Fax: +251-58-111-7678, E-mail: beletebiadigo@yahoo.com, ORCID: https://orcid.org/0000-0002-4468-0242

Financial support: None. Conflict of interest: None. 
(dysbiosis) has been associated with pathologies, such as inflammatory bowel disease and obesity. ${ }^{8-10}$

The gut microbiota is now considered a separate organ that is involved in regulating several physiological pathways by influencing different functions of the host. ${ }^{11}$ Among these regulatory actions, the effect of gut microbes on the energy metabolism and the driving force in the pathogenesis of metabolic diseases, particularly obesity, is an important factor. Intestinal microbes have established a mutually beneficial link with their host by modulating the gut motility, intestinal barrier homeostasis to prevent potentially harmful bacteria from causing damage to the tissues, nutrient absorption, and fat distribution. ${ }^{12-14}$

Evidence shows that gut microbiota play a critical role in the development of obesity and T2DM. ${ }^{15-17}$ Studies have suggested that obese people with IR can be characterized by an altered composition of gut microbiota, predominantly an elevated Firmicutes/Bacteroidetes ratio compared to healthy people. $^{18,19}$

T2DM patients showed an altered intestinal microbiota, which is characterized by a decrease in the Bacteroidetes/ Firmicutes ratio and some functional bacteria (e.g., Bifidobacteria) with an increase in various opportunistic pathogens as well as some endotoxins-producing gram negative bacteria ${ }^{20-22}$ that alter the host energy metabolism through a specific polysaccharide utilization loci mechanism. ${ }^{22}$ Moreover, the accumulation of gut-derived bacterial inflammatory molecules (e.g., lipopolysaccharide [LPS], peptidoglycans and flagellin) in the intestine is thought to hasten the inflammation in T2DM. ${ }^{23,24}$

The human intestine harbors hundreds of trillions of bacteria, as well as bacteriophage particles, viruses, fungi and archaea, which constitute a complex and dynamic ecosystem with which an individual lives in symbiosis throughout lifetime. ${ }^{25}$ Host genetics is believed to contribute to the profile of the gut microbiome, living conditions including dietary habits, exposure to xenobiotic (such as drugs, toxicants, and additives) or stresses (such as surgery and infections) modulate the gut microbiota, occasionally for a limited period of time due to the resilience of this ecosystem. ${ }^{26}$ The gut microbiota characterizes an environmental factor of T2DM that was abandoned in the past because of the complexity of its analysis ${ }^{27}$ and to a lack of understanding of the mechanisms underlying the interactions between gut microbes and host metabolism. Therefore, microbiota is comprised of microorganisms that not only monitor the body homeostasis but are also the driving force in the pathogenesis of metabolic disease. This review summarizes the current knowledge concerning the role of gut microbiota in T2DM pathogenesis and its related complications and provides novel insights and reliable information on the gut microbiota as a therapeutic role and potential intervention strategy for the management of DM complications.

\section{GUT MICROBIOTA ROLE IN DM PATHOGENESIS}

Microorganisms in the GIT are referred to collectively as the gut microbiota. Qin et al. ${ }^{28}$ reported that the gut microbiota mainly encompasses four main phyla: Actinobacteria, Bacteroidetes, Firmicutes, and Proteobacteria. These are vital in the host metabolism and physiology regulation. Mika et al. $^{29}$ discussed the microbiota during early childhood, which include Actinobacteria predominantly of the genus Bifidobacterium, that dominate the gut microbiota of breastfed infants. Through time, the microbiota gains a variety of new strains influenced by changes in diet and by disease, finally beginning to resemble the adult composition. ${ }^{29}$ In addition, Prakash et al. ${ }^{30}$ indicated that physical exercise could also modulate the gut microbiota.

The GIT is the primary site of interaction between the host immune system and microorganisms, both symbiotic and pathogenic. ${ }^{31}$ The gut microbiota act as a protective mediator during pathological conditions. The Gl system represents one of the largest boundaries between the human internal microenvironment and the external world. This system harbors trillions of commensal bacteria existing in symbiosis with the host. Intestinal bacteria play a vital role in maintaining the systemic and intestinal immune and metabolic homeostasis because of their effects on nutrient absorption and immune development and function. ${ }^{32} \mathrm{Xu}$ et al. ${ }^{33}$ reported that the composition of the gut microbial communities vary along the GIT between individuals as the dietary lifestyle and nutritional status of the individual varies.

Many factors affect the gut microbiota composition and its structural functions, such as the intestinal architecture regulation; gut permeability; immune system and barrier function; and protective and metabolic functions, such as breast feeding, genetic background, antibiotics, diet, lifestyle, bacteria in the amniotic fluid, and delivery procedure. ${ }^{34}$ In addition to 
digestion, the gut microbiota is important for maintaining the optimal state of host health, but it is also associated with the pathogenesis of numerous metabolic diseases, such as obesity, ${ }^{35,36}$ diabetes, ${ }^{22,37}$ chronic kidney disease, ${ }^{38,39}$ and atherosclerosis, ${ }^{40-42}$ and intestinal diseases, such as inflammatory bowel diseases ${ }^{43}$ and colorectal cancer. ${ }^{4,45}$ Sabatino et al. ${ }^{46}$ reported that dysbiosis is associated with endotoxemia and chronic inflammation, with a disturbance of the intestinal barrier and the depletion of useful bacteria that lead to different complications.

The hosts and their microbiomes develop symbiotic relationships through interactive evolutionary processes that mutually benefit both. Xie et al. ${ }^{47}$ reported that resident symbionts regulate the host metabolism in many ways, integrating physiological homeostasis, immune-inflammatory signaling, and energy compliance. Carbohydrates are the primary sources of energy for both the human host and their microbes.

The gut microbiome can also act as an endocrine organ that translates the nutritional cues to hormone-like signals to control host physiology and diseases. The gut microbiome also interacts with the inflammatory, cardiovascular system via metabolic signaling pathways, which can lead to chronic systemic inflammation that is supported by different scholars. Brown et al., ${ }^{48}$ Boulangé et al.. ${ }^{49}$ Kelly et al.. ${ }^{50}$ and Paras et al. ${ }^{51}$ indicated that the gut microbiome has interactions with systemic diseases, including obesity, diabetes, hepatopathy, rheumatoid arthritis, cancer, and cardiovascular diseases.

\section{PATHOPHYSIOLOGY OF THE GUT MICROBIOTA ON DIABETICS}

Over the past decade, there has been an increasing focus on gut microbiota as an important factor in the development of inflammatory disease in both humans and animals. ${ }^{52,53}$ The GIT microbiome interrelates with the host nutrition, environment, and host genetics for the development of obesity-related metabolic disorders. Turnbaugh et al. ${ }^{54}$ reported that GIT microbial dysbiosis enhances energy harvesting and the expression of an obese phenotype. A change in the Bacteroidetes/Firmicutes ratio is associated with the higher expression of microbial genes that encode the enzymes related to carbohydrate metabolism. In this report, the microbiomes of obese persons differ from those of lean in- dividuals and are characterized by a lower prevalence of phylum Bacteroidetes and a higher prevalence of phylum Firmicutes. ${ }^{54}$ The microbiome of the gut activates changes in the intestine tight junction proteins and alkaline phosphatase activity in the gut environment, which may increase the gut permeability and lead to the pathogenesis of $\mathrm{IR}^{13}$

Cani et al. ${ }^{55}$ attempted to explain the mechanism of how the gut bacteria induces the inflammatory state of obesity via the activity of LPS, which is a component of gram-negative bacterial cell walls that can activate the inflammatory process by binding to the CD14 toll-like receptor-4 (TLR-4) complex at the surface of innate immune cells. LPS is a ligand of TLR-4. ${ }^{55}$ The importance of the TLR-4 pathways in metabolic disease was confirmed by the finding that a deletion of TLR- 4 prevented high-fat diet-induced IR. ${ }^{56}$ The Bacteroidetes/ Firmicutes ratio is an environmental factor that provides genetic material for an increased capacity to harvest energy from the diet. ${ }^{57}$ Murphy et al. ${ }^{58}$ reported that the higher energy harvest promotes lipogenesis and increases the number and size of lipid droplets in the extra intestinal tissues. Most patients suffering from this metabolic syndrome have extreme fat accumulation, which suggests that the dyslipidemia is an important etiological factor of the syndrome. ${ }^{58}$

In the liver, butyrate can be metabolized into glutamate, glutamine, and acetoacetate. Acetoacetate is an important fuel source for intestinal cells. As an intestinal nutrient, butyrate stimulates the regeneration of intestinal cells to repair the intestinal mucosa, initiates the differentiation and apoptosis of normal intestinal cells, stimulates the production of intestinal mucin glycoprotein, and fortifies the defensive outcome of the mucous layer. On the other hand, acetate can increase the total cholesterol, and propionate increases glucose in the blood and reduces the hypercholesterolemia response caused by acetate. ${ }^{59}$

Alterations in the microbiome fermentation profile change the gut permeability and energy homeostasis, which causes endotoxemia, low-grade inflammation, and obesity. Poor energy homeostasis leads to hyperglycemia and hyperlipidemia, which may lead to obesity and ultimately IR. ${ }^{60} \mathrm{~T} 2 \mathrm{DM}$ in humans was reported to be co-related to a lower abundance of butyrate-producing microbes and an increased abundance of Lactobacillus species. ${ }^{22,37}$

The intestinal bacteria generate short chain fatty acids (SCFAs) by fermenting dietary carbohydrates that humans can- 
not digest themselves. Martin et al. ${ }^{61}$ reported that germ-free mice are devoid of SCFAs, indicating the importance of the gut microbiota for SCFA production in the intestine. The role of the GIT microbiome in the development of the metabolic syndrome that leads to DM pathogenesis has been described. ${ }^{60}$ Microbial dysbiosis harms the intestinal wall integrity and allows the translocation of toxins from the gut lumen to the systemic circulation. This endotoxemia leads to low-grade inflammation, autoimmunity, and oxidative stress that may lead to beta cell destruction or IR. ${ }^{60}$

\section{COMPLICATIONS OF THE GUT MICROBIOTA DYSBIOSIS ON T2DM}

The harmful effects of hyperglycemia are separated into micro-vascular complications (diabetic nephropathy, neuropathy, and retinopathy) and macro-vascular complications (coronary artery disease, peripheral arterial disease, and stroke) ${ }^{62}$ A disturbance in the composition of the microbiota is strongly related to the incidence of inflammatory diseases, supporting the key role of a commensal microbiota in host homeostasis. ${ }^{63}$ Beli et al. ${ }^{64}$ reported that intermittent fasting prevents diabetic retinopathy in mice by restructuring the microbiota towards species producing tauroursodeoxycholate and subsequent retinal protection by activating the Takeda G-protein-coupled receptor 5 . Moreover, mice on an intermittent fasting regimen displayed significantly longer survival and a reduction in the diabetic retinopathy endpoints, including acellular capillaries and leukocyte infiltration. ${ }^{64}$ Kanbay et al. ${ }^{65}$ and Mafra and Fouque ${ }^{66}$ reported that the gut microbiota interacts closely with the inflammatory, renal, cardiovascular, and endocrine systems via metabolic, humoral, and neural signaling pathways. They also indicated that favorable modification of the composition and function of the gut microbiome represents an alluring therapeutic target for the prevention and treatment of chronic kidney disease. ${ }^{65,66}$ For example, a disruption of the normal physiology of the microbiota in the gut causes DM complications. New insights into the role of the gut microbiota in DM complications could lead to the development of therapeutic strategies using probiotics to prevent and treat these metabolic disorders. ${ }^{34}$

\section{GUT MICROBIOTA AS THERAPEUTIC ALTERNATIVES FOR DM}

The gut microbial diversity, metalloproteins encoding gene expression in gut bacterial species, and glycemic index are improved during a metformin treatment for T2DM. ${ }^{67}$ Recently, metformin has been proposed as an adjuvant treatment for cancer, $^{68}$ as a treatment for gestational diabetes, and for the prevention of T2DM in pre-diabetic individuals. ${ }^{69}$ Stades et al. $^{70}$ suggested metformin, which is currently recommended by the practice guidelines as the first line therapy for the majority of patients with T2DM.

On the other hand, bariatric surgery alters the composition and diversity of the gut microbiota in humans, rats and mice significantly. ${ }^{71-73}$ An alteration of microbial dysbiosis by supplementation with prebiotics improved bifidobacterium abundance, which is significantly and positively associated with improved glucose tolerance and inflammation in prebiotic treated mice. ${ }^{74}$ Buchwald et al. $^{75}$ reported that the clinical and laboratory manifestations of T2DM are improved in a majority of patients after bariatric surgery. Strategies for the treatment of gut microbiota dysbiosis through the supplementation or ingestion of live beneficial bacteria (probiotics) have been suggested. ${ }^{76}$

The reintegration of intestinal microbiota by the mean of pre- or pro-biotic is a treatment alternative for T2DM patients. ${ }^{77}$ Probiotics are live, natural microorganisms that are given orally to deliberate health benefits to the host. ${ }^{78}$ The interest in managing the microbiota composition to increase the potentially beneficial aspects has increased. The prebiotic approach dictates that non-viable food components fermented specifically in the colon by native bacteria have a positive value, e.g., Bifidobacteria and Lactobacilli. $^{77,79}$

Fecal microbiota transplantation (FMT) also increased the survival rate of irradiated animals, raised the peripheral white blood cell counts, and improved the GIT function and intestinal epithelial integrity in irradiated male and female mice. Cui et al. ${ }^{80}$ reported that the transplantation of fecal microbiota from healthy mice to irradiated mice upgraded the GIT function and epithelial integrity of the small intestines in a sex-dependent fashion to ameliorate radiation-induced toxicity. Bang et al., ${ }^{81}$ who examined FMT for refractory and recurrent Clostridium difficile infection $(\mathrm{CDI})$, reported that FMT is a safe, well-tolerated, and highly effective treatment for re- 
fractory/recurrent CDIs. Moreover, the authors also expect that FMT will be used widely to treat refractory/recurrent CDI.

\section{CONCLUSIONS AND RECOMMENDATIONS}

Epidemiological studies indicated the association of the gut microbiota and T2DM. In many studies, an association between the disturbance of gut microbiota and an increased incidence rate of T2DM was indicated. The energy metabolism is of particular interest because it has been proposed to be a driving force in the pathogenesis of metabolic diseases, particularly obesity that in turn induces T2DM. On the other hand, the supplementation of prebiotics is an important mechanism for the rehabilitation of gut microbiota and for the harmony of body homeostasis.

In general, the early detection and screening of new onset T2DM patients for gut microbiota dysbiosis are recommended for the management of patients with T2DM. Understanding the mechanisms of the relationship between DM and the gut microbiota is important for improving long-term survival. Future research should examine the development of novel preventive and therapeutic strategies for gut microbiota alterations among T2DM patients.

\section{REFERENCES}

1. Definition, diagnosis and classification of diabetes mellitus and its complications : report of a WHO consultation. Part 1, diagnosis and classification of diabetes mellitus. [Internet]. Geneva: World Health Organization; c1999 [cited 2018 Oct 20]. Avaliable from: http://www.who.int/iris/handle/10665/66040

2. American Diabetes Association. Standards of medical care in diabetes. Diabetes Care 2014;37(Suppl 1):S14-S80.

3. Sigurdardóttir AK. Self-care in diabetes: Model of factors affecting self-care. J Clin Nurs 2005;14:301-314.

4. Longo DL, Kasper DL, Jameson JL, Fauci AS, Hauser SL, LoscalzoJ. Harrison's principles of internal medicine. 18th ed. New York (PA): McGraw Hill Professional, 2011.

5. International Diabetes Federation. IDF diabetes atlas. 7th ed. Brussels: International Diabetes Federation, 2015.

6. International Diabetes Federation, IDF diabetes atlas. 6th ed. Brussels: International Diabetes Federation, 2013.

7. UK prospective diabetes study 6 . Complications in newly diagnosed type 2 diabetic patients and their association with different clinical and biochemical risk factors. Diabetes Res 1990; 13:1-11.

8. Ley RE, Turnbaugh PJ, Klein S, Gordon Jl. Microbial ecology: human gut microbes associated with obesity. Nature 2006;444: 1022-1023.

9. Marteau P, Lepage P, Mangin I, et al. Review article: gut flora and inflammatory bowel disease. Aliment Pharmacol Ther 2004;20 Suppl 4:18-23.

10. Swidsinski A, Weber J, Loening-Baucke V, Hale LP, Lochs H. Spatial organization and composition of the mucosal flora in patients with inflammatory bowel disease. J Clin Microbiol 2005; 43:3380-3389.

11. Cani PD. Metabolism in 2013: the gut microbiota manages host metabolism. Nat Rev Endocrinol 2014;10:74-76.

12. Kashyap PC, Marcobal A, Ursell LK, et al. Complex interactions among diet, gastrointestinal transit, and gut microbiota in humanized mice. Gastroenterology 2013;144:967-977.

13. Everard A, Cani PD. Diabetes, obesity and gut microbiota. Best Pract Res Clin Gastroenterol 2013;27:73-83.

14. Zhao L. The gut microbiota and obesity: from correlation to causality. Nat Rev Microbiol 2013;11:639-647.

15. Ley RE, Bäckhed F, Turnbaugh P, Lozupone CA, Knight RD, Gordon JI. Obesity alters gut microbial ecology. Proc Natl Acad Sci U S A 2005;102:11070-11075.

16. Cani PD, Bibiloni R, Knauf C, et al. Changes in gut microbiota control metabolic endotoxemia-induced inflammation in high-fat diet-induced obesity and diabetes in mice. Diabetes 2008;57: 1470-1481.

17. Cani PD, Possemiers S, Van de Wiele T, et al. Changes in gut microbiota control inflammation in obese mice through a mechanism involving GLP-2-driven improvement of gut permeability. Gut 2009;58:1091-1103.

18. Tilg $\mathrm{H}$, Kaser A. Gut microbiome, obesity, and metabolic dysfunction. J Clin Invest 2011;121:2126-2132.

19. Tremaroli V, Bäckhed F. Functional interactions between the gut microbiota and host metabolism. Nature 2012;489:242-249.

20. Wu X, Ma C, Han L, et al. Molecular characterisation of the faecal microbiota in patients with type II diabetes. Curr Microbiol 2010; 61:69-78.

21. Larsen N, Vogensen FK, van den Berg FW, et al. Gut microbiota in human adults with type 2 diabetes differs from non-diabetic adults. PLoS One 2010;5:e9085.

22. Qin J, Li Y, Cai Z, et al. A metagenome-wide association study of gut microbiota in type 2 diabetes. Nature 2012;490:55-60.

23. Kootte RS, Vrieze A, Holleman F, et al. The therapeutic potential of manipulating gut microbiota in obesity and type 2 diabetes mellitus. Diabetes Obes Metab 2012;14:112-120.

24. Delzenne NM, Cani PD. Gut microbiota and the pathogenesis of insulin resistance. Curr Diab Rep 2011;11:154-159.

25. Hoffmann C, Dollive S, Grunberg S, et al. Archaea and fungi of the human gut microbiome: correlations with diet and bacterial residents. PLoS One 2013;8:e66019.

26. Goodrich JK, Waters JL, Poole AC, et al. Human genetics shape the gut microbiome. Cell 2014;159:789-799.

27. Delzenne NM, Cani PD, Everard A, Neyrinck AM, Bindels LB. Gut microorganisms as promising targets for the management of type 2 diabetes. Diabetologia 2015;58:2206-2217.

28. Qin J, Li R, Raes J, et al. A human gut microbial gene catalogue established by metagenomic sequencing. Nature 2010;464: 59-65.

29. Mika A, Van Treuren W, González A, Herrera JJ, Knight R, Fleshner $M$. Exercise is more effective at altering gut microbial composi- 
tion and producing stable changes in lean mass in juvenile versus adult male F344 rats. PLoS One 2015;10:e0125889.

30. Prakash S, Rodes L, Coussa-Charley M, Tomaro-Duchesneau C. Gut microbiota: next frontier in understanding human health and development of biotherapeutics. Biologics 2011;5:71-86.

31. Round JL, Mazmanian SK. The gut microbiota shapes intestinal immune responses during health and disease. Nat Rev Immunol 2009;9:313-323.

32. Needell JC, Zipris D. The role of the intestinal microbiome in type 1 diabetes pathogenesis. Curr Diab Rep 2016;16:89.

33. Xu J, Mahowald MA, Ley RE, et al. Evolution of symbiotic bacteria in the distal human intestine. PLoS Biol 2007;5:e156.

34. Blandino G, Inturri R, Lazzara F, Di Rosa M, Malaguarnera L. Impact of gut microbiota on diabetes mellitus. Diabetes Metab 2016;42:303-315.

35. Ridaura VK, Faith JJ, Rey FE, et al. Gut microbiota from twins discordant for obesity modulate metabolism in mice. Science 2013;341:1241214.

36. Turnbaugh PJ, Hamady M, Yatsunenko T, et al. A core gut microbiome in obese and lean twins. Nature 2009;457:480-484.

37. Karlsson FH, Tremaroli V, Nookaew I, et al. Gut metagenome in European women with normal, impaired and diabetic glucose control. Nature 2013;498:99-103.

38. Wang F, Zhang P, Jiang H, Cheng S. Gut bacterial translocation contributes to microinflammation in experimental uremia. Dig Dis Sci 2012;57:2856-2862.

39. Mishima E, Fukuda S, Shima H, et al. Alteration of the intestinal environment by lubiprostone is associated with amelioration of adenine-induced CKD. J Am Soc Nephrol 2015;26:1787-1794.

40. Tang WH, Wang Z, Levison BS, et al. Intestinal microbial metabolism of phosphatidylcholine and cardiovascular risk. N Engl J Med 2013;368:1575-1584.

41. Koeth RA, Wang Z, Levison BS, et al. Intestinal microbiota metabolism of L-carnitine, a nutrient in red meat, promotes atherosclerosis. Nat Med 2013;19:576-585.

42. Wang Z, Roberts AB, Buffa JA, et al. Non-lethal inhibition of gut microbial trimethylamine production for the treatment of atherosclerosis. Cell 2015;163:1585-1595.

43. Molodecky NA, Soon IS, Rabi DM, et al. Increasing incidence and prevalence of the inflammatory bowel diseases with time, based on systematic review. Gastroenterology 2012;142:46-54.e42.

44. Belcheva A, Irrazabal T, Robertson SJ, et al. Gut microbial metabolism drives transformation of MSH2-deficient colon epithelial cells. Cell 2014;158:288-299.

45. Schulz MD, Atay Ç, Heringer J, et al. High-fat-diet-mediated dysbiosis promotes intestinal carcinogenesis independently of obesity. Nature 2014;514:508-512.

46. Sabatino A, Regolisti G, Cosola C, Gesualdo L, Fiaccadori E. Intestinal microbiota in type 2 diabetes and chronic kidney disease. Curr Diab Rep 2017;17:16.

47. Xie B, Waters MJ, Schirra HJ. Investigating potential mechanisms of obesity by metabolomics. J Biomed Biotechnol 2012;2012: 805683.

48. Brown JM, Hazen SL. The gut microbial endocrine organ: bacterially derived signals driving cardiometabolic diseases. Annu Rev Med 2015;66:343-359.
49. Boulangé CL, Neves AL, Chilloux J, Nicholson JK, Dumas ME. Impact of the gut microbiota on inflammation, obesity, and metabolic disease. Genome Med 2016;8:42.

50. Kelly TN, Bazzano LA, Ajami NJ, et al. Gut microbiome associates with lifetime cardiovascular disease risk profile among bogalusa heart study participants. Circ Res 2016;119:956-964.

51. Paras TM, Percival F, McQuade E. The gut microbiome influences arthritis development in IIJ mice. J Immunol 2016;196(Suppl 1):118.16.

52. Hooper LV, Littman DR, Macpherson AJ. Interactions between the microbiota and the immune system. Science 2012;336: 1268-1273.

53. Bleich A, Hansen AK. Time to include the gut microbiota in the hygienic standardisation of laboratory rodents. Comp Immunol Microbiol Infect Dis 2012;35:81-92.

54. Turnbaugh PJ, Bäckhed F, Fulton L, Gordon Jl. Diet-induced obesity is linked to marked but reversible alterations in the mouse distal gut microbiome. Cell Host Microbe 2008;3:213-223.

55. Cani PD, Neyrinck AM, Fava F, et al. Selective increases of Bifidobacteria in gut microflora improve high-fat-diet-induced diabetes in mice through a mechanism associated with endotoxaemia. Diabetologia 2007;50:2374-2383.

56. Shi H, Kokoeva MV, Inouye K, Tzameli I, Yin H, Flier JS. TLR4 links innate immunity and fatty acid-induced insulin resistance. J Clin Invest 2006;116:3015-3025.

57. Turnbaugh PJ, Ley RE, Mahowald MA, Magrini V, Mardis ER, Gordon JI. An obesity-associated gut microbiome with increased capacity for energy harvest. Nature 2006;444:1027-1031.

58. Murphy EF, Cotter PD, Healy S, et al. Composition and energy harvesting capacity of the gut microbiota: relationship to diet, obesity and time in mouse models. Gut 2010;59:1635-1642.

59. Brown AJ, Goldsworthy SM, Barnes AA, et al. The orphan G protein-coupled receptors GPR41 and GPR43 are activated by propionate and other short chain carboxylic acids. J Biol Chem 2003;278:11312-11319.

60. Sohail MU, Althani A, Anwar H, Rizzi R, Marei HE. Role of the gastrointestinal tract microbiome in the pathophysiology of diabetes mellitus. J Diabetes Res 2017;2017:9631435.

61. Martin FP, Wang Y, Sprenger N, et al. Probiotic modulation of symbiotic gut microbial-host metabolic interactions in a humanized microbiome mouse model. Mol Syst Biol 2008;4:157.

62. Fowler MJ. Microvascular and macrovascular complications of diabetes. Clinical Diabetes 2008;26:77-82.

63. David LA, Maurice CF, Carmody RN, et al. Diet rapidly and reproducibly alters the human gut microbiome. Nature 2014;505: 559-563.

64. Beli E, Yan Y, Moldovan L, et al. Restructuring of the gut microbiome by intermittent fasting prevents retinopathy and prolongs survival in db/db mice. Diabetes 2018;69:1867-1879.

65. Kanbay M, Onal EM, Afsar B, et al. The crosstalk of gut microbiota and chronic kidney disease: role of inflammation, proteinuria, hypertension, and diabetes mellitus. Int Urol Nephrol 2018;50: 1453-1466.

66. Mafra D, Fouque D. Gut microbiota and inflammation in chronic kidney disease patients. Clin Kidney J 2015;8:332-334.

67. Wu H, Esteve E, Tremaroli V, et al. Metformin alters the gut micro- 
biome of individuals with treatment-naive type 2 diabetes, contributing to the therapeutic effects of the drug. Nat Med 2017; 23:850-858

68. Quinn BJ, Kitagawa H, Memmott RM, Gills JJ, Dennis PA. Repositioning metformin for cancer prevention and treatment. Trends Endocrinol Metab 2013;24:469-480.

69. Gui J, Liu Q, Feng L. Metformin vs insulin in the management of gestational diabetes: a meta-analysis. PLoS One 2013;8: e64585.

70. Stades AM, Heikens JT, Erkelens DW, Holleman F, Hoekstra JB. Metformin and lactic acidosis: cause or coincidence? A review of case reports. J Intern Med 2004;255:179-187.

71. Ryan KK, Tremaroli V, Clemmensen C, et al. FXR is a molecular target for the effects of vertical sleeve gastrectomy. Nature 2014;509:183-188.

72. Tremaroli V, Karlsson F, Werling M, et al. Roux-en-Y gastric bypass and vertical banded gastroplasty induce long-term changes on the human gut microbiome contributing to fat mass regulation. Cell Metab 2015;22:228-238.

73. Yang PJ, Yang WS, Nien HC, et al. Duodenojejunal bypass leads to altered gut microbiota and strengthened epithelial barriers in rats. Obes Surg 2016;26:1576-1583.

74. Cani PD, Amar J, Iglesias MA, et al. Metabolic endotoxemia ini- tiates obesity and insulin resistance. Diabetes 2007;56: 1761-1772.

75. Buchwald H, Estok R, Fahrbach K, et al. Weight and type 2 diabetes after bariatric surgery: systematic review and meta-analysis. Am J Med 2009;122:248-256.e5.

76. Conlon MA, Bird AR. The impact of diet and lifestyle on gut microbiota and human health. Nutrients 2014;7:17-44.

77. Roberfroid MB. Inulin-type fructans: functional food ingredients. J Nutr 2007;137(11 Suppl):2493S-2502S.

78. Hill C, Guarner F, Reid G, et al. Expert consensus document. The international scientific association for probiotics and prebiotics consensus statement on the scope and appropriate use of the term probiotic. Nat Rev Gastroenterol Hepatol 2014;11: 506-514

79. Roberfroid M. Prebiotics: the concept revisited. J Nutr 2007; 137(3 Suppl 2):830S-837S.

80. Cui M, Xiao H, Li Y, et al. Faecal microbiota transplantation protects against radiation-induced toxicity. EMBO Mol Med 2017;9: 448-461.

81. Bang BW, Park JS, Kim HK, et al. Fecal microbiota transplantation for refractory and recurrent clostridium difficile infection: a case series of nine patients. Korean J Gastroenterol 2017;69: 226-231. 